\title{
Advanced Lung Cancer Inflammation Index is a Prognostic Factor of Patients with Small-Cell Lung Cancer Following Surgical Resection
}

This article was published in the following Dove Press journal: Cancer Management and Research

\author{
Zhonghui $\mathrm{Hu}^{1,2, *}$ \\ Wenbo Wu (1D) ${ }^{1,3, *}$ \\ Xiaopeng Zhang ${ }^{\prime}$ \\ Ping $\mathrm{Li}^{4}, *$ \\ Hua Zhang' \\ Huien Wang' \\ Wenfei Xue \\ Zhiguo Chen ${ }^{5}$ \\ Qingtao Zhao (D) \\ Guochen Duan (D) ${ }^{1,5}$ \\ 'Department of Thoracic Surgery, Hebei \\ General Hospital, Shijiazhuang, People's \\ Republic of China; ${ }^{2}$ Graduate School, \\ Hebei Medical University, Shijiazhuang, \\ People's Republic of China; ${ }^{3}$ Graduate \\ School, Hebei North University, \\ Zhangjiakou, People's Republic of China; \\ ${ }^{4}$ Department of Thoracic Surgery, Zigong \\ First People's Hospital, Zigong, People's \\ Republic of China; ${ }^{5}$ Department of \\ Thoracic Surgery, Hebei Children's \\ Hospital, Shijiazhuang, People's Republic \\ of China
}

*These authors contributed equally to this work

Correspondence: Guochen Duan Hebei Children's Hospital, No. 133 Jianhua South Street, Yuhua District, Shijiazhuang, 050000, People's Republic of China

Tel +863II 13380703

Email duanguoc@126.com

Qingtao Zhao

Hebei General Hospital, No. 384 Heping

West Road, Xinhua District, Shijiazhuang,

050000, People's Republic of China

Tel +8631185988756

Email taolII82004@I63.com
Purpose: Advanced lung cancer inflammation index (ALI) has been shown to predict overall survival (OS) in advanced non small-cell lung cancer (NSCLC), small-cell lung cancer (SCLC) and operable NSCLC. However, there were no studies of the correlation between ALI and operable SCLC. Therefore, this study is aimed to explore the relationship between ALI and the prognosis of operable SCLC.

Patients and Methods: A total of 48 patients with SCLC who underwent surgery at Hebei General Hospital and Zigong First People's Hospital were screened between 2016 and 2020. ALI was calculated as follows: body mass index (BMI, $\left.\mathrm{kg} / \mathrm{m}^{2}\right) \times$ serum albumin (ALB, g/dL)/ neutrophil to lymphocyte ratio (NLR). Receiver operating characteristic (ROC) curve was used to determine the optimal cutoff value of ALI. Patients were divided into two groups according to the cutoff point of ALI: low ALI group with ALI $<48.2$ and high ALI group with $\mathrm{ALI} \geq 48.2$. Kaplan-Meier and Cox regression analysis were performed to assess the potential prognostic factors associated with OS.

Results: The optimal cutoff value of ALI was determined as 48.2. The low ALI group displayed more adverse clinical characteristics and poorer survival rates. Multivariate analysis revealed that ALI and Charlson comorbidity index (CCI) were significantly correlated with OS.

Conclusion: Low ALI was correlated with poor prognosis in patients with SCLC who underwent surgery. Preoperative ALI might serve as a potential prognostic marker for patients with operable SCLC.

Keywords: ALI, SCLC, surgery, prognosis

\section{Introduction}

Lung cancer remains the leading cause of cancer-related deaths in China, and smallcell lung cancer (SCLC) accounts for approximately $15 \%$ of all cases. ${ }^{1,2}$ SCLC, highly responsive to initial chemotherapy and radiotherapy, is highly aggressive and poor in prognosis. ${ }^{3,4}$ Therefore, it is important to explore accurate prognostic factors for SCLC. The dichotomized staging system and TNM Classification of Malignant Tumors (TNM) are the most important predictors of overall survival (OS). ${ }^{5}$ In addition, a number of clinical indicators such as gender, age, smoking status and performance status (PS) are reported to be related to prognosis in patients with SCLC. ${ }^{4,6,7}$ There is increasing evidence that inflammation status is correlated with

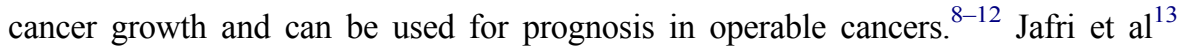
developed a prognostic index based on systemic inflammation called advanced lung 
cancer inflammation index (ALI), which was calculated as body mass index (BMI, $\left.\mathrm{kg} / \mathrm{m}^{2}\right) \times$ serum albumin (ALB, g/ $\mathrm{dL}$ )/neutrophil to lymphocyte ratio (NLR). Although the prognostic effect of ALI has been tested in patients with SCLC, esophageal cancer, and malignant lymphoma, ${ }^{14-16}$ the prognostic significance of ALI in operable SCLC has not yet been investigated. Operable SCLC refer to patients with SCLC that can be cured by local resection. In this study, we explored whether ALI is associated with the prognosis of patients with operable SCLC.

\section{Patients and Methods}

From January 2016 to August 2020, a total of 48 patients with SCLC who underwent curative surgery at Hebei General Hospital and Zigong First People's Hospital were enrolled. Curative surgery refers to local resection of lung tissue of SCLC patients to reach a cure. No patients received preoperative chemoradiotherapy. Patients with acute or chronic inflammatory disease during the preoperative period were excluded. All patients were pathologically diagnosed as SCLC with no clinical evidence of infection or other inflammatory conditions and no other malignancies.

End point of assessment was patient OS, which was defined as the time from operation to the time of death from any cause. For subjects who had missed their followup visits prior to death, the last follow-up was counted as the time of death. The clinicopathological variables including age, gender, height, weight, smoking status, PS and Charlson comorbidity index (CCI) were recorded by the electronic medical record system. Laboratory parameters including C-creative protein (CRP), neutrophil count, lymphocyte count, white blood cell count (WBC), red blood cell count (RBC), platelet count (PLT), monocyte count (MONO), eosinophil count (EO), basophil count (BASO), ALB and lactate dehydrogenase (LDH) were performed before operation. The preoperative serum carcinoembryonic antigen (CEA), squamous cell carcinoma antigen (SCC), cytokeratin-19 fragment (CYFRA 21-1), progastrin releasing peptide (Pro-GRP) and neuron-specific enolase (NSE) levels were measured in all patients by enzyme immunoassay in single laboratory at both hospitals. The median postoperative follow-up was 26 months. Ethical Committee of Hebei General Hospital and Zigong First People's Hospital approved this retrospective study. Informed consent was waived due to the retrospective nature of the study. We confirm the confidentiality of the data maintained and compliance with the "Declaration of Helsinki".

ALI was calculated by the following formula: BMI $\left(\mathrm{kg} / \mathrm{m}^{2}\right) \times$ ALB $(\mathrm{g} / \mathrm{dL}) / \mathrm{NLR}^{13}$ Cutoff value for ALI, ALB, WBC, RBC, PLT, MONO, EO, BASO, NLR, platelet to lymphocyte ratio (PLR) and lymphocyte to monocyte ratio (LMR) were determined using receiver operating characteristic (ROC) curve analysis to estimate optimal sensitivity, specificity, and the area under the curve (AUC) for prediction of death from all causes. Pearson correlation, Chi-square test and Fisher exact test were used to compare continuous and categorical variables. Cumulative cancer-specific survival curves after surgery were calculated using the Kaplan-Meier method, and differences were assessed using Log rank test. The Cox proportional hazard model was used to evaluate the predictive power of potential prognostic variables, and the hazard ratios (HR) estimated from the Cox analysis reported as relative risks with corresponding 95\% confidence intervals. Statistical analyses were performed using the IBM SPSS statistics software program, version 21.0 (IBM Corporation, Armonk, NY, USA). $\mathrm{P}<0.05$ indicated statistical significance.

\section{Results}

In total, 48 patients with SCLC receiving surgical resection were analyzed, according to available clinical information and baseline laboratory parameters (Table 1). The median age was 60.5 years (range: 27 to 80 years). Thirty-four patients $(70.8 \%)$ were male and 28 patients $(58.3 \%)$ were current or ever smokers. Thirty-three patients $(68.8 \%)$ scored

Table I Clinical Characteristics of Patients

\begin{tabular}{|l|l|l|}
\hline & Baseline & No. \\
\hline Age & $\begin{array}{l}<65 \text { years } \\
\geq 65 \text { years }\end{array}$ & 31 \\
& Male & 17 \\
\hline Gender & Female & 34 \\
& Never & 14 \\
\hline Smoking & Current/ever & 23 \\
& $<18.5$ & 25 \\
\hline BMI & $\geq 18.5$ to $<25$ & 2 \\
& $\geq 25$ & 23 \\
& $0-1$ & 23 \\
\hline PS & 2 & 33 \\
& 2 & 15 \\
\hline
\end{tabular}

(Continued) 
Table I (Continued).

\begin{tabular}{|c|c|c|}
\hline & Baseline & No. \\
\hline \multirow[t]{3}{*}{$\mathrm{CCl}$} & 0 & 19 \\
\hline & $1-2$ & 13 \\
\hline & $\geq 3$ & 16 \\
\hline \multirow[t]{2}{*}{ ALB (g/dL) } & $<4.13$ & 23 \\
\hline & $\geq 4.13$ & 25 \\
\hline \multirow[t]{2}{*}{ WBC $\left(\times 10^{9} / \mathrm{L}\right)$} & $<6.47$ & 23 \\
\hline & $\geq 6.47$ & 25 \\
\hline \multirow[t]{2}{*}{$\operatorname{RBC}\left(\times 10^{12} / L\right)$} & $<4.30$ & 15 \\
\hline & $\geq 4.30$ & 33 \\
\hline \multirow[t]{2}{*}{ PLT $\left(\times 10^{9} / \mathrm{L}\right)$} & $<231.50$ & 24 \\
\hline & $\geq 231.50$ & 24 \\
\hline \multirow[t]{2}{*}{ MONO $\left(\times 10^{9} / \mathrm{L}\right)$} & $<0.38$ & 31 \\
\hline & $\geq 0.38$ & 17 \\
\hline \multirow[t]{2}{*}{$\mathrm{EO}\left(\times 10^{9} / \mathrm{L}\right)$} & $<0.12$ & 28 \\
\hline & $\geq 0.12$ & 20 \\
\hline \multirow[t]{2}{*}{ BASO $\left(\times 10^{9} / \mathrm{L}\right)$} & $<0.05$ & 38 \\
\hline & $\geq 0.05$ & 10 \\
\hline \multirow[t]{2}{*}{ NLR } & $<2.08$ & 20 \\
\hline & $\geq 2.08$ & 28 \\
\hline \multirow[t]{2}{*}{ LMR } & $<10.86$ & 46 \\
\hline & $\geq 10.86$ & 2 \\
\hline \multirow[t]{2}{*}{ PLR } & $<124.27$ & 22 \\
\hline & $\geq 124.27$ & 26 \\
\hline \multirow[t]{2}{*}{ Pro-GRP } & Normal & 3 \\
\hline & High & 21 \\
\hline \multirow[t]{2}{*}{ NSE } & Normal & 28 \\
\hline & High & 20 \\
\hline \multirow[t]{2}{*}{ Postoperative chemotherapy } & Yes & 33 \\
\hline & No & 15 \\
\hline
\end{tabular}

Abbreviations: $\mathrm{BMI}$, body mass index; PS, performance status; $\mathrm{CCl}$, Charlson comorbidity index; $A L B$, albumin count; WBC, white blood cell count; RBC, red blood cell count; PLT, platelet count; MONO, monocyte count; EO, eosinophil count; BASO, basophil count; NLR, neutrophil to lymphocyte ratio; LMR, lymphocyte to monocyte ratio; PLR, platelet to lymphocyte ratio; ALI, advanced lung cancer inflammation index; CEA, carcinoembryonic antigen; LDH, lactate dehydrogenase; SCC, squamous cell carcinoma antigen; CYFRA 2I-I, cytokeratin-19 fragment; Pro-DRP, pro-gastrin releasing peptide; NSE, neuron-specific enolase; CRP, C-reactive protein.

PS as 0 or 1 while 15 patients $(31.3 \%)$ scored CCI as 0 . Thirty-three patients $(68.8 \%)$ received postoperative chemotherapy (Etoposide-based combination chemotherapy). Nine patients had lung cancer recurrence, and 6 of them died. In total, 15 patients died during the observation period.

The median value of ALI was 46.8 (16.8 to 77.4). The optimal cutoff point shown by ROC curve analysis of ALI for the layering of OS in SCLC was determined to be 48.2 (Figure 1A). Optimal cutoff points of RBC $\left(\times 10^{12} / \mathrm{L}\right)$, WBC $\left(\times 10^{9} / \mathrm{L}\right)$, PLT $\left(\times 10^{9} / \mathrm{L}\right)$, MONO $\left(\times 10^{9} / \mathrm{L}\right)$, EO $\left(\times 10^{9} / \mathrm{L}\right)$, BASO $\left(\times 10^{9} / \mathrm{L}\right), \mathrm{LMR}, \mathrm{PLR}, \mathrm{NLR}$ and ALB $(\mathrm{g} / \mathrm{dL})$ were 4.30, 6.47, 231.50, 0.38, 0.12, 0.45, 10.86, 124.27, 2.08 and 4.13 , respectively (Figure $1 \mathrm{~B}$ ). Patients were divided into two groups according to the ALI value based on the cutoff point: low ALI group with $\operatorname{ALI}<48.2(n=25)$ and high ALI group with $A L I \geq 48.2 \quad(n=23)$. The relationship between baseline characteristics and ALI are shown in Table 2. Comparison of postoperative survival curves of the two groups showed a significant difference in the rates of patient survival (Figure 2, $\mathrm{P}<0.05$ ). Patients with high ALI had longer OS than those in low ALI group (34 vs 19 months, $\mathrm{P}<0.05)$.

The low ALI group displayed more adverse clinical characteristics. OS, PFS, PS, CCI, LDH, CRP, NLR and CYFRA21-1 were significantly different between the two groups $(\mathrm{P}<0.05)$. In univariable analysis of OS, lowALI, smoking, $\mathrm{PS}=2, \mathrm{CCI} \geq 3$, high $\mathrm{MONO}$, high $\mathrm{EO}$, high BASO and high LDH were significant factors for poor survival $(\mathrm{P}<0.05)$ (Table 3$)$. In multivariable analyses using Cox hazard model (Table 3), $\mathrm{CCI} \geq 3$ and low ALI were independent predictors of poor prognosis $(\mathrm{P}<0.05)$. In addition, MONO and EO might be independent prognostic factors for operable SCLC $(\mathrm{p}<0.1)$.

\section{Discussion}

Inflammation and immune response can affect angiogenesis and cellular proliferation, which play key roles in carcinogenesis. ${ }^{8,17}$ Epidemiological observation has implicated that systemic inflammation is important in cancer etiology as inflammatory markers were evident in tumor microenvironment, which indicated the prognostic significance of inflammation in different cancers. Furthermore, recent studies have shown the correlation between systematic inflammation and poor cancer outcomes. ${ }^{6,12,14,18,19}$ Inflammation is widely involved in multiple pathological conditions including lymphocytopenia, neutrophilia, thrombocytosis and so on. ${ }^{20}$ Previous studies have shown that absolute inflammatory cell counts in peripheral blood (neutrophils, WBC, lymphocytes and MONO) and ratios based on these cell counts (NLR, PLR and LMR) may provide valuable information in predicting the prognosis of patients with malignances including NSCLC. ${ }^{21-23}$ Therefore, these inflammatory parameters may also predict the prognosis of operable SCLC. 
A

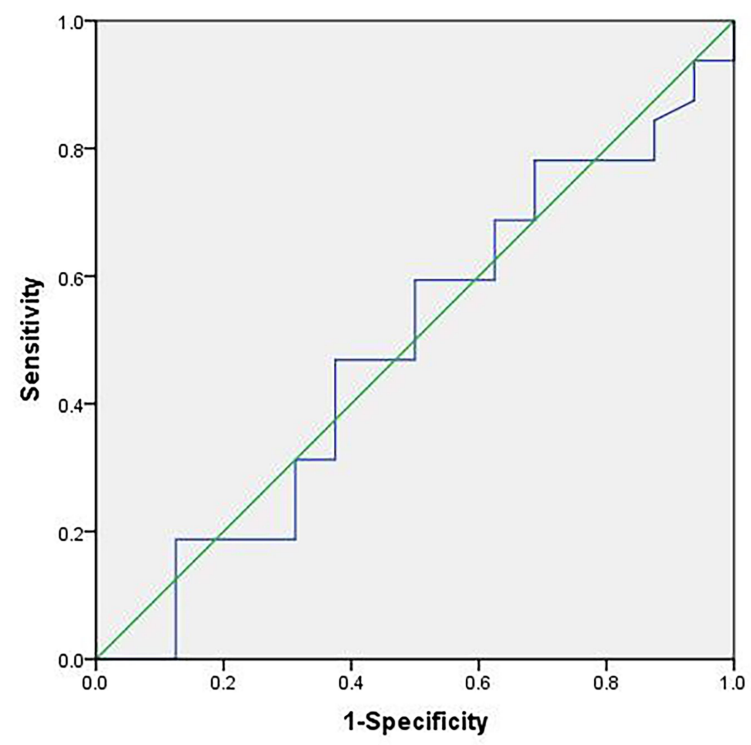

B

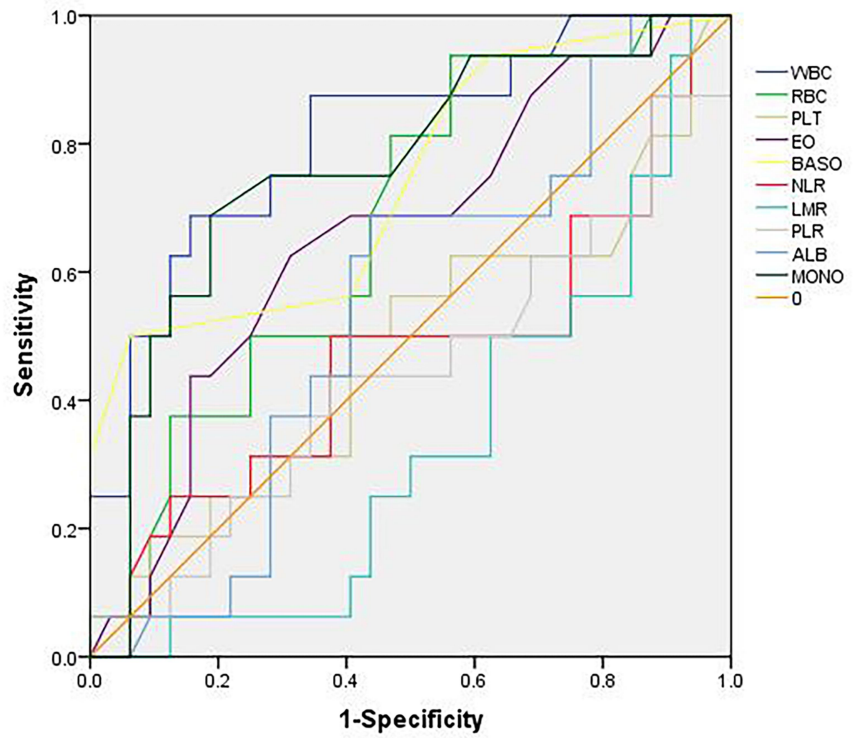

Figure I Receiver operating characteristic (ROC) curves. (A) shows the ROC curve of advanced lung cancer inflammation index (ALI). The maximum Youden index is 0.094, corresponding optimal cutoff value of ALI is 48.2. (B) shows the ROC curves of red blood cell count (RBC), white blood cell count (WBC), platelet count (PLT), monocyte count (MONO), eosinophil count (EO), basophil count (BASO), lymphocyte to monocyte ratio (LMR), platelet to lymphocyte ratio (PLR), neutrophil to lymphocyte ratio (NLR) and serum albumin (ALB). Maximum Youden indexes are as follows: 0.375 for RBC, 0.53 I for WBC, 0.094 for PLT, 0.500 for MONO, 0.313 for EO, 0.438 for BASO, 0.063 for LMR, 0.063 for PLR, 0.125 for NLR and 0.250 for ALB. Optimal cutoff points of RBC $\left(\times 10^{12} / \mathrm{L}\right), \mathrm{WBC}\left(\times 10^{9} / \mathrm{L}\right), \mathrm{PLT}\left(\times 10^{9} / \mathrm{L}\right), \mathrm{MONO}\left(\times 10^{9} / \mathrm{L}\right)$, EO $\left(\times 10^{9} / \mathrm{L}\right)$, BASO $\left(\times 10^{9} / \mathrm{L}\right), \mathrm{LMR}, \mathrm{PLR}, \mathrm{NLR}$ and ALB $(\mathrm{g} / \mathrm{dL})$ are $4.30,6.47,231.50,0.38,0.12,0.45,10.86,124.27,2.08$ and 4.13.

Neutrophils, remodeling the extracellular matrix and releasing reactive oxygen species or nitric oxide, are thought to participate in the process of tumor proliferation and metastasis. ${ }^{24}$ Platelets can affect the metastatic potential of tumor cells by secreting cellular growth factors which can stimulate tumor angiogenesis, thereby contributing to the stable adhesion and transmigration of tumor cells, promoting tumor stroma formation and tumor cell metastasis. ${ }^{25,26}$ Therefore, an elevated neutrophil count and platelet count may be correlated with a poor prognosis of patients with cancer. ${ }^{27}$ Lymphocytes play important roles in tumor immune surveillance, killing cancer cells and regulating the cancer progression. The cytotoxic activity of lymphocytes and their induction of apoptosis in tumor cells can control tumor growth. ${ }^{28,29}$ Decrease in lymphocytes may count as a biomarker of poor outcome in patients with terminal cancer. Moreover, monocytes can facilitate the progression and dissemination of tumor cells. They can be recruited to the tumor microenvironment to promote tumor cell growth and survival. The differentiation of monocytes can be induced into tumor-associated macrophages, which can weaken the anti-tumor immune response and stimulate the migration and metastasis of tumor cells. ${ }^{17,30}$ Peripheral monocyte levels were found to be negatively associated with prognosis in patients with various types of cancer. ${ }^{27,31,32}$

In addition, the significant prognostic effect of NLR, PLR and LMR on OS in cancer patients has been reported by various studies, ${ }^{33-36}$ suggesting that elevated NLR, PLR and reduced MLR may predict poor prognosis of SCLC. In this study, NLR, WBC, PLT, PLR and LMR have no significant effect on OS of operable SCLC patients. Univariable analysis showed that MONO has prognostic value while multivariable analysis showed no significant value. The results might due to small sample size in our study, and tumors in early-stage rarely show obvious inflammation and migration.

Tumor-associated blood eosinophilia, accounts for $1-7 \%$ of all clinical eosinophilia's diagnoses, ${ }^{37}$ was described in various tumors. ${ }^{38,39}$ In addition, basophils were reported to be accessory cells exerting clinically relevant tumor-promoting functions in pancreatic cancer. ${ }^{40}$ However, these findings are not frequently observed in clinical practice. Our study demonstrated the prognostic value of EO and BASO while multivariable analysis showed no independent value. The mechanisms of the two factors in tumors may be related to immune response, which need further investigation. 
Table 2 Relationship Between Patient Characteristics and ALI

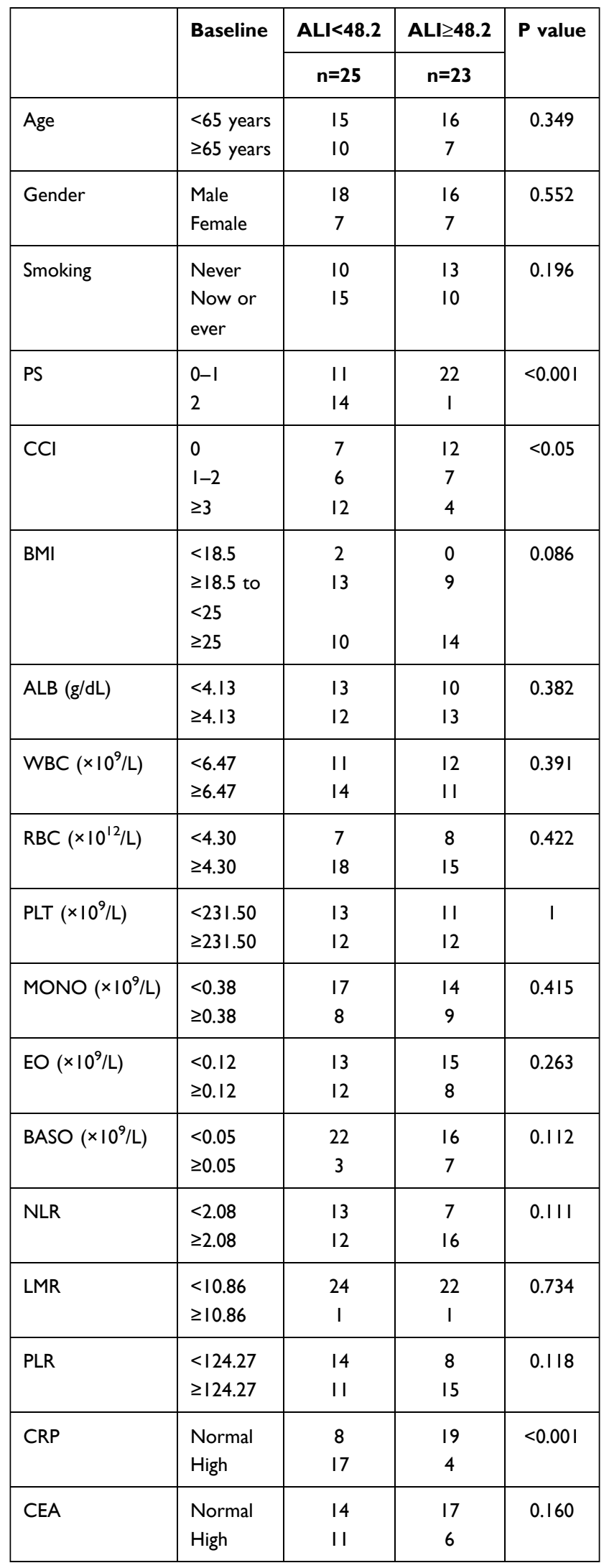

(Continued)
Table 2 (Continued).

\begin{tabular}{|c|c|c|c|c|}
\hline & \multirow[t]{2}{*}{ Baseline } & \multirow{2}{*}{$\begin{array}{c}\text { ALI }<48.2 \\
n=25\end{array}$} & \multirow{2}{*}{$\begin{array}{c}A L I \geq 48.2 \\
n=23\end{array}$} & \multirow[t]{2}{*}{$P$ value } \\
\hline & & & & \\
\hline \multirow[t]{2}{*}{ LDH } & Normal & 13 & 19 & $<0.05$ \\
\hline & High & 12 & 4 & \\
\hline \multirow[t]{2}{*}{ SCC } & Normal & 12 & 11 & 0.592 \\
\hline & High & 6 & 6 & \\
\hline \multirow[t]{2}{*}{ CYFRA2I-I } & Normal & 9 & 16 & $<0.05$ \\
\hline & High & 7 & 16 & \\
\hline \multirow[t]{2}{*}{ Pro-GRP } & Normal & 1 & 2 & 0.249 \\
\hline & High & 15 & 6 & \\
\hline \multirow[t]{2}{*}{ NSE } & Normal & 14 & 14 & $0.48 I$ \\
\hline & High & 9 & 11 & \\
\hline \multirow{2}{*}{$\begin{array}{l}\text { Postoperative } \\
\text { chemotherapy }\end{array}$} & Yes & 9 & 6 & 0.335 \\
\hline & No & 16 & 17 & \\
\hline \multirow[t]{2}{*}{ Living status } & Live & 17 & 15 & 0.540 \\
\hline & Death & 8 & 8 & \\
\hline
\end{tabular}

Abbreviations: $\mathrm{BMI}$, body mass index; PS, performance status; $\mathrm{CCl}$, Charlson comorbidity index; ALB, albumin; WBC, white blood cell; RBC, red blood cell; PLT, platelet; MONO, monocyte; EO, eosinophil; BASO, basophil; NLR, neutrophil to lymphocyte ratio; LMR, lymphocyte to monocyte ratio; PLR, platelet to lymphocyte ratio; ALI, advanced lung cancer inflammation index; CEA, carcinoembryonic antigen; LDH, lactate dehydrogenase; SCC, squamous cell carcinoma antigen; CYFRA 2I-I, cytokeratin-19 fragment; Pro-GRP, pro-gastrin releasing peptide; NSE, neuron-specific enolase; CRP, C-reactive protein.

Cancer cachexia is a multi-factorial syndrome, considered as the clinical consequence of interactions between tumor, metabolism and inflammatory factors, impairing quality of life and response to therapy. ${ }^{41}$ Though cachexia rarely appear in early-stage oncologic patients, estimation of body composition is of widely considerable importance. Previous study has indicated that body weight loss in advanced cancer may increase the risk of mortality. ${ }^{42}$ BMI and ALB have been demonstrated to be important parameters for evaluation of nutritional status. ${ }^{43-45}$ The two factors were reported to be closely linked to survival in patients with not only advanced lung cancer, but also resected NSCLC. ${ }^{44-47}$ Therefore, they might be prognostic factors in operable SCLC patients. Tomita et $\mathrm{al}^{46}$ reported that hypoalbuminaemia might be an important marker of inflammation in addition to malnutrition in patients with operable NSCLC, and ALI might be a useful prognostic marker in patients with resected NSCLC. Zhou et $\mathrm{al}^{6}$ demonstrated the correlation between systemic inflammatory 


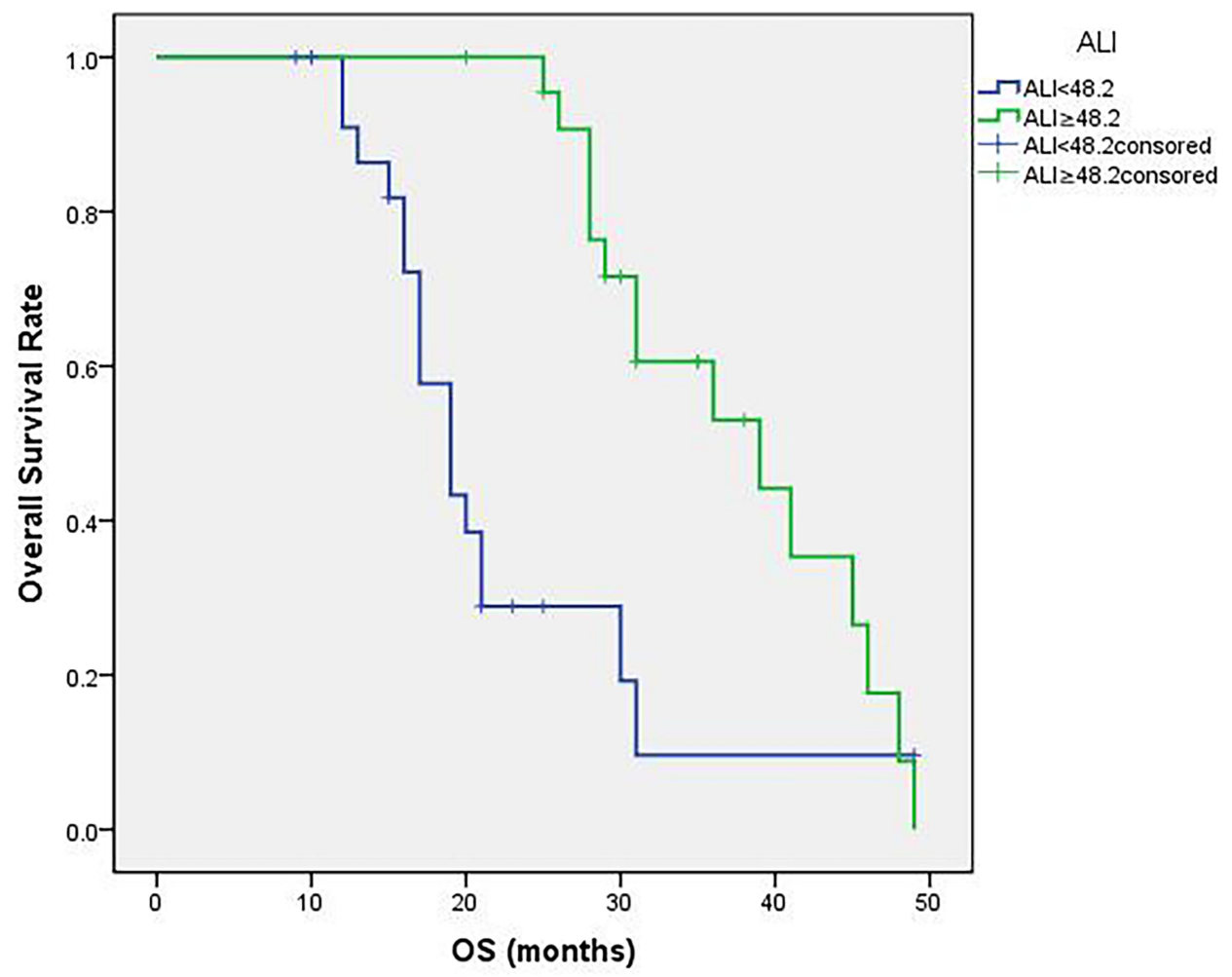

Figure 2 Patient overall survival (OS) curves following surgery. The curves indicate that high ALI group (ALI $\geq 48.2)$ shows more survival rates and longer survival time $(\mathrm{P}<0.05)$.

response and cancer-specific survival in patients with SCLC. Since inflammation and malnutrition factors play important roles in many kinds of tumor including SCLC, it is likely that ALI, calculated from BMI, ALB and NLR, could be a prognostic marker in operable SCLC patients.

We investigated SCLC cases with radical resection in the present study and found the correlation between ALI and prognosis. Our findings indicate the importance of malnutrition and inflammatory status in prognosis of not only advanced lung cancer, but also early-stage operable SCLC. We determined a cutoff value of 48.2 for ALI with ROC curve analysis. In previous studies, different cutoff values such as 31.1 by Kim and colleagues, ${ }^{48} 47.0$ by Zhou et $\mathrm{al}^{49}$ and 19.5 by $\mathrm{He}$ et $\mathrm{al}^{14}$ have been reported in patients with small-cell lung cancer. Jafri et $\mathrm{al}^{13}$ demonstrated a cutoff value of 18 in patients with advanced NSCLC, while Tomita et $\mathrm{al}^{50}$ reported that of 37.66 in patients with resected NSCLC and Kobayashi et $\mathrm{al}^{51}$ that of 22.2 in early-stage NSCLC. Our analysis resulted in a cutoff value within the range of those reported in other studies. OS, PFS, NLR, serum CYFRA21-1 level, PS and alive status were different between patients with high and low ALI. The analysis results strongly indicate that ALI could be a prognostic factor for operable SCLC patients.

Our data suggest that preoperative ALI, along with smoking, PS, CCI and $\mathrm{LDH}$, is a prognostic factor for operable SCLC. Furthermore, ALI and CCI could be independent prognostic factors. Other inflammation indicators we evaluated are not of statistical significance for prognosis, which may due to small sample size and early-stage cancer that do not show obvious inflammation with significant increase. Radical resection is associated with significantly longer OS for early SCLC. Studies showed five-year OS for patients with clinical stages I and II after resection to be 51\% and $25 \% .{ }^{52}$ However, due to poor OS in SCLC patients, postoperative treatment is suggested. Previous studies have shown the effect of targeted therapy ${ }^{53}$ and immunotherapy ${ }^{54}$ on reducing postoperative recurrence rates. By studying the prognostic factors of SCLC patients, we can better assess the prognostic risk and give appropriate postoperative adjuvant treatments to effectively extend patients' lifetime.

\section{Limitations}

The study has limitations in the following aspects. It is a retrospective study and we did not select postoperative 
Table 3 Univariable and Multivariable Analysis

\begin{tabular}{|c|c|c|c|c|c|c|c|c|}
\hline & \multicolumn{4}{|c|}{ Univariable Analysis } & \multicolumn{4}{|c|}{ Multivariable Analysis } \\
\hline & \multirow[t]{2}{*}{$P$ value } & \multirow[t]{2}{*}{ HR } & \multicolumn{2}{|c|}{$95 \% \mathrm{Cl}$} & \multirow[t]{2}{*}{$P$ value } & \multirow[t]{2}{*}{ HR } & \multicolumn{2}{|c|}{$95 \% \mathrm{Cl}$} \\
\hline & & & LL & UL & & & LL & UL \\
\hline ALI & 0.031 & 0.309 & 0.107 & 0.896 & 0.009 & 0.035 & 0.003 & 0.425 \\
\hline Gender & 0.070 & 53.193 & 0.722 & 3918.139 & 0.952 & $260,285.584$ & 0 & \\
\hline Age & 0.505 & 1.454 & 0.484 & 4.370 & & & & \\
\hline Smoking & 0.005 & 6.280 & 1.750 & 22.534 & 0.538 & 0.492 & 0.051 & 4.704 \\
\hline PS & 0.019 & 0.276 & 0.094 & 0.809 & & & & \\
\hline $\mathrm{CCl}=0$ & 0.011 & & & & 0.137 & & & \\
\hline $\mathrm{CCl}=\mathrm{I}-2$ & 0.936 & 0.943 & 0.225 & 3.949 & 0.310 & 3.644 & 0.301 & 44.150 \\
\hline $\mathrm{CCl} \geq 3$ & 0.006 & 7.011 & 1.739 & 28.267 & 0.046 & 9.355 & 1.037 & 84.381 \\
\hline ALB & 0.675 & 1.236 & 0.458 & 3.336 & & & & \\
\hline WBC & 0.669 & 0.806 & 0.299 & 2.170 & & & & \\
\hline $\mathrm{RBC}$ & 0.093 & 43.222 & 0.534 & 3495.431 & 0.955 & $292,578.176$ & 0 & \\
\hline PLT & 0.487 & $\mathrm{I} .42 \mathrm{I}$ & 0.528 & 3.826 & & & & \\
\hline MONO & 0.001 & 6.590 & 2.067 & 21.015 & 0.073 & 0.225 & 0.044 & 1.151 \\
\hline EO & 0.023 & 3.562 & 1.194 & 10.632 & 0.094 & 5.371 & 0.750 & 38.493 \\
\hline BASO & 0.012 & 3.711 & 1.335 & 10.314 & 0.475 & 0.567 & 0.120 & 2.685 \\
\hline NLR & 0.069 & 0.384 & 0.137 & 1.079 & 0.323 & 2.090 & 0.484 & 9.022 \\
\hline LMR & 0.567 & 0.045 & 0 & 1813.598 & & & & \\
\hline PLR & 0.393 & 0.650 & 0.242 & $\mathrm{I} .747$ & & & & \\
\hline CEA & 0.745 & 1.184 & 0.427 & 3.289 & & & & \\
\hline LDH & 0.046 & 2.867 & 1.017 & 8.079 & 0.826 & 0.783 & 0.089 & $6.88 I$ \\
\hline SCC & 0.556 & 1.533 & 0.370 & 6.359 & & & & \\
\hline CYFRA2I-I & 0.127 & 2.284 & 0.790 & 6.599 & & & & \\
\hline NSE & 0.152 & 2.074 & 0.765 & 5.624 & 0.329 & 0.319 & 0.032 & 3.156 \\
\hline BMI $<18.5$ & 0.243 & & & & & & & \\
\hline BMI[I8.5,25) & 0.797 & 0.745 & 0.079 & 7.037 & & & & \\
\hline $\mathrm{BMI} \geq 25$ & 0.114 & 2.383 & 0.812 & 6.991 & & & & \\
\hline CRP & 0.926 & 1.053 & 0.357 & 3.103 & & & & \\
\hline Pro-GRP & 0.960 & 0.946 & 0.110 & 8.136 & & & & \\
\hline Postoperative chemotherapy & 0.644 & 0.775 & 0.263 & 2.283 & & & & \\
\hline
\end{tabular}

Abbreviations: ALI, advanced lung cancer inflammation index; PS, performance status; $\mathrm{CCl}$, Charlson comorbidity index; ALB, albumin; WBC, white blood cell; RBC, red blood cell; PLT, platelet; MONO, monocyte; EO, eosinophil; BASO, basophil; NLR, neutrophil to lymphocyte ratio; LMR, lymphocyte to monocyte ratio; PLR, platelet to lymphocyte ratio; CEA, carcinoembryonic antigen; LDH, lactate dehydrogenase; SCC, squamous cell carcinoma antigen; CYFRA 2 I-I, cytokeratin-19 fragment; NSE, neuron-specific enolase; BMI, body mass index; CRP, C-reactive protein; ProGRP, pro-gastrin releasing peptide.

data of ALI. Thus, there is potential of selection bias. Multivariate analysis showed that ALI could be an independent prognostic factor. Therefore, a large-scale multicenter prospective validation study is required to establish more reliable and independent findings.

\section{Conclusion}

Inflammation plays a vital role in cancer prognosis. Preoperative ALI, smoking, PS, CCI and LDH may be prognostic factors for SCLC patients receiving radical surgery. ALI, along with CCI, may be an independent and effective prognostic factor for patients with operable
SCLC, which can be adapted in the clinical practice to stratify SCLC patients for future trials.

\section{Acknowledgments}

This work was supported by the Hebei Medical Applied Technology tracking Project (No. G2018008), the Key projects of Medical Science Research in Hebei Province (No. 20180151) and the Scientific Research Program of traditional Chinese Medicine (No. 2019139).

\section{Disclosure}

The authors report no conflicts of interest in this work. 


\section{References}

1. Chen W, Zheng R, Baade PD, et al. Cancer statistics in China, 2015. CA Cancer J Clin. 2016;66(2):115-132.

2. Byers LA, Rudin CM. Small cell lung cancer: where do we go from here? Cancer. 2015;121(5):664-672.

3. van Meerbeeck JP, Fennell DA, De Ruysscher DK. Small-cell lung cancer. Lancet. 2011;378(9804):1741-1755. doi:10.1016/S01406736(11)60165-7

4. Torre LA, Bray F, Siegel RL, et al. Global cancer statistics, 2012. $C A$ Cancer J Clin. 2015;65(2):87-108. doi:10.3322/caac.21262

5. Zelen M. Keynote address on biostatistics and data retrieval. Cancer Chemother Rep 3. 1973;4(2):31-42.

6. Zhou T, Hong S, Hu Z, et al. A systemic inflammation-based prognostic scores (mGPS) predicts overall survival of patients with small-cell lung cancer. Tumour Biol. 2015;36(1):337-343. doi:10.10 07/s13277-014-2623-4

7. Fruh M, De Ruysscher D, Popat S, et al. Small-cell lung cancer (SCLC): ESMO clinical practice guidelines for diagnosis, treatment and follow-up. Ann Oncol. 2013;24(Suppl 6):vi99-105. doi:10.1093/ annonc/mdt 178

8. Coussens LM, Werb Z. Inflammation and cancer. Nature. 2002;420 (6917):860-867. doi:10.1038/nature01322

9. McMillan DC. The systemic inflammation-based Glasgow prognostic score: a decade of experience in patients with cancer. Cancer Treat Rev. 2013;39(5):534-540. doi:10.1016/j.ctrv.2012.08.003

10. Grange JM, Krone B, Mastrangelo G. Infection, inflammation and cancer. Int $J$ Cancer. 2011;128(9):2240-2241. doi:10.1002/ijc.25533

11. Tomita M, Shimizu T, Ayabe T, et al. Elevated preoperative inflammatory markers based on neutrophil-to-lymphocyte ratio and C-reactive protein predict poor survival in resected non-small cell lung cancer. Anticancer Res. 2012;32(8):3535-3538.

12. Proctor MJ, Morrison DS, Talwar D, et al. A comparison of inflammation-based prognostic scores in patients with cancer: a Glasgow inflammation outcome Study. Eur J Cancer. 2011;47 (17):2633-2641. doi:10.1016/j.ejca.2011.03.028

13. Jafri SH, Shi R, Mills G. Advance lung cancer inflammation index (ALI) at diagnosis is a prognostic marker in patients with metastatic non-small cell lung cancer (NSCLC): a retrospective review. $B M C$ Cancer. 2013;13(1):158. doi:10.1186/1471-2407-13-158

14. He X, Zhou T, Yang Y, et al. Advanced lung cancer inflammation index, a new prognostic score, predicts outcome in patients with small-cell lung cancer. Clin Lung Cancer. 2015;16(6):e165-171. doi:10.1016/j.cllc.2015.03.005

15. Feng JF, Huang Y, Chen QX. A new inflammation index is useful for patients with esophageal squamous cell carcinoma. Onco Targets Ther. 2014;7:1811-1815. doi:10.2147/OTT.S68084

16. Park YH, Yi HG, Lee MH, et al. Prognostic value of the pretreatment advanced lung cancer inflammation index (ALI) in diffuse large B cell lymphoma patients treated with R-CHOP chemotherapy. Acta Haematol. 2017;137(2):76-85. doi:10.1159/000452991

17. Mantovani A, Allavena P, Sica A, et al. Cancer-related inflammation. Nature. 2008;454(7203):436-444. doi:10.1038/nature07205

18. Balkwill F, Mantovani A. Inflammation and cancer: back to virchow? Lancet. 2001;357(9255):539-545. doi:10.1016/S0140-6736(00)04046-0

19. Balkwill F, Charles KA, Mantovani A. Smoldering and polarized inflammation in the initiation and promotion of malignant disease. Cancer Cell. 2005;7(3):211-217. doi:10.1016/j.ccr.2005.02.013

20. Chen Y, Zhang L, Liu WX, et al. Prognostic significance of preoperative anemia, leukocytosis and thrombocytosis in Chinese women with epithelial ovarian cancer. Asian Pac J Cancer Prev. 2015;16 (3):933-939. doi:10.7314/APJCP.2015.16.3.933

21. Zhao QT, Yang Y, Xu S, et al. Prognostic role of neutrophil to lymphocyte ratio in lung cancers: a meta-analysis including 7054 patients. Onco Targets Ther. 2015;8:2731-2738. doi:10.2147/OTT. S90875
22. Charles KA, Harris BD, Haddad CR, et al. Systemic inflammation is an independent predictive marker of clinical outcomes in mucosal squamous cell carcinoma of the head and neck in oropharyngeal and non-oropharyngeal patients. BMC Cancer. 2016;16(1):124. doi:10.11 86/s12885-016-2089-4

23. Sekine K, Kanda S, Goto Y, et al. Change in the lymphocyte-tomonocyte ratio is an early surrogate marker of the efficacy of nivolumab monotherapy in advanced non-small-cell lung cancer. Lung Cancer. 2018;124:179-188. doi:10.1016/j.lungcan.2018.08.012

24. Uribe-Querol E, Rosales C. Neutrophils in cancer: two sides of the same coin. J Immunol Res. 2015;2015:983698. doi:10.1155/2015/ 983698

25. Wang Q, Li Z, Sun L, et al. Platelets enhance the ability of bone-marrow mesenchymal stem cells to promote cancer metastasis. Onco Targets Ther. 2018;11:8251-8263. doi:10.2147/ OTT.S181673

26. Kono SA, Heasley LE, Doebele RC, et al. Adding to the mix: fibroblast growth factor and platelet-derived growth factor receptor pathways as targets in non-small cell lung cancer. Curr Cancer Drug Targets. 2012;12(2):107-123. doi:10.2174/156800912799095144

27. Valero C, Pardo L, Lopez M, et al. Pretreatment count of peripheral neutrophils, monocytes, and lymphocytes as independent prognostic factor in patients with head and neck cancer. Head Neck. 2017;39 (2):219-226. doi:10.1002/hed.24561

28. Eerola AK, Soini Y, Paakko P. A high number of tumor-infiltrating lymphocytes are associated with a small tumor size, low tumor stage, and a favorable prognosis in operated small cell lung carcinoma. Clin Cancer Res. 2000;6(5):1875-1881.

29. Hiraoka K, Miyamoto M, Cho Y, et al. Concurrent infiltration by $\mathrm{CD} 8+\mathrm{T}$ cells and CD4+ $\mathrm{T}$ cells is a favourable prognostic factor in non-small-cell lung carcinoma. Br J Cancer. 2006;94(2):275-280. doi:10.1038/sj.bjc.6602934

30. Peng LS, Zhang JY, Teng YS, et al. Tumor-associated monocytes/ macrophages impair NK-cell function via TGFbeta1 in human gastric cancer. Cancer Immunol Res. 2017;5(3):248-256. doi:10.1158/23266066.CIR-16-0152

31. Wang YQ, Zhu YJ, Pan JH, et al. Peripheral monocyte count: an independent diagnostic and prognostic biomarker for prostate cancer - a large Chinese cohort study. Asian J Androl. 2017;19(5):579-585. doi:10.4103/1008-682X.186185

32. Han L, Jia Y, Song Q, et al. Prognostic significance of preoperative absolute peripheral monocyte count in esophageal squamous cell carcinoma. Dis Esophagus. 2016;29(7):740-746. doi:10.1111/dote. 12401

33. Kim EY, Lee JW, Yoo HM, et al. The platelet-to-lymphocyte ratio versus neutrophil-to-lymphocyte ratio: which is better as a prognostic factor in gastric cancer? Ann Surg Oncol. 2015;22(13):4363-4370. doi:10.1245/s10434-015-4518-z

34. Shao N, Cai Q. High pretreatment neutrophil-lymphocyte ratio predicts recurrence and poor prognosis for combined small cell lung cancer. Clin Transl Oncol. 2015;17(10):772-778. doi:10.1007/s120 94-015-1289-8

35. Hirahara N, Matsubara T, Kawahara D, et al. Prognostic significance of preoperative inflammatory response biomarkers in patients undergoing curative thoracoscopic esophagectomy for esophageal squamous cell carcinoma. Eur J Surg Oncol. 2017;43(2):493-501. doi:10. 1016/j.ejso.2016.11.018

36. Pan YC, Jia ZF, Cao DH, et al. Preoperative lymphocyte-to-monocyte ratio (LMR) could independently predict overall survival of resectable gastric cancer patients. Medicine (Baltimore). 2018;97(52): e13896. doi:10.1097/MD.0000000000013896

37. Costello R, O'Callaghan T, Sébahoum G. Eosinophils and antitumour response. Rev Med Interne. 2005;26(6):479-484. doi:10.1016/j. revmed.2005.02.013

38. Dellon AL, Hume RB, Chretien PB. Eosinophilia in bronchogenic carcinoma. N Eng lJ Med. 1974;291:207-208. 
39. Divack DM, Janovski NA. Eosinophilia encountered in female genital organs. Am J Obstet Gynecol. 1962;84(6):761-763. doi:10.1016/ 0002-9378(62)90028-5

40. De Monte L, Wörmann S, Brunetto E, et al. Basophil recruitment into tumor-draining lymph nodes correlates with Th2 inflammation and reduced survival in pancreatic cancer patients. Cancer Res. 2016;76 (7):1792-1803. doi:10.1158/0008-5472.CAN-15-1801-T

41. Fearon K, Strasser F, Anker SD, et al. Definition and classification of cancer cachexia: an international consensus. Lancet Oncol. 2011;12 (5):489-495. doi:10.1016/S1470-2045(10)70218-7

42. McMillan DC. An inflammation-based prognostic score and its role in the nutrition-based management of patients with cancer. Proc Nutr Soc. 2008;67(3):257-262. doi:10.1017/S0029665108007131

43. Jin Y, Zhao L, Peng F. Prognostic impact of serum albumin levels on the recurrence of stage I non-small cell lung cancer. Clinics (Sao Paulo). 2013;68(5):686-693. doi:10.6061/clinics/2013(05)17

44. Nakagawa T, Toyazaki T, Chiba N, et al. Prognostic value of body mass index and change in body weight in postoperative outcomes of lung cancer surgery. Interact Cardiovasc Surg. 2016;23(4):560-566. doi:10.1093/icvts/ivw175

45. Tewari N, Martin-Ucar AE, Black E, et al. Nutritional status affects long term survival after lobectomy for lung cancer. Lung Cancer. 2007;57(3):389-394. doi:10.1016/j.lungcan.2007.03.017

46. Tomita M, Ayabe T, Chosa E, et al. Prognostic significance of preand postoperative Glasgow prognostic score for patients with non small cell lung cancer. Anticancer Res. 2014;34(6):3137-3140.

47. Yotsukura M, Ohtsuka T, Kaseda K, et al. Value of the Glasgow prognostic score as a prognostic factor in resectable non-small cell lung cancer. J Thorac Oncol. 2016;11(8):1311-1318. doi:10.1016/j. jtho.2016.04.029
48. Kim EY, Kim N, Kim YS, et al. Prognostic significance of modified advanced lung cancer inflammation index (ALI) in patients with small cell lung cancer - comparison with original ALI. PLoS One. 2016;11(10):e0164056. doi:10.1371/journal.pone.0164056

49. Zhou T, Zhao Y, Zhao S, et al. Comparison of the prognostic value of systemic inflammation response markers in small cell lung cancer patients. J Cancer. 2019;10(7):1685-1692. doi:10.7150/jca.29319

50. Tomita M, Ayabe T, Nakamura K. The advanced lung cancer inflammation index is an independent prognostic factor after surgical resection in patients with nonsmall-cell lung cancer. Interact Cardiovasc Thorac Surg. 2018;26(2):288-292. doi:10.1093/icvts/ivx329

51. Kobayashi S, Karube Y, Inoue T, et al. Advanced lung cancer inflammation index predicts outcomes of patients with pathological stage IA lung adenocarcinoma following surgical resection. Ann Thorac Cardiovasc Surg. 2019;25(2):87-94. doi:10.5761/atcs.oa.18-00158

52. Combs SE, Hancock JG, Boffa DJ, et al. Bolstering the case for lobectomy in stages I, II, and IIIA small-cell lung cancer using the national cancer data base. J Thorac Oncol. 2015;10(2):316-323. doi:10.1097/JTO.0000000000000402

53. Roudi R, Madjd Z, Ebrahimi M, et al. Evidence for embryonic stem-like signature and epithelial-mesenchymal transition features in the spheroid cells derived from lung adenocarcinoma. Tumour Biol. 2016;37(9):11843-11859. doi:10.1007/s13277-016-5041-y

54. zhu J, Li R, Tiselius E, et al. Immunotherapy (excluding checkpoint inhibitors) for stage I to III non-small cell lung cancer treated with surgery or radiotherapy with curative intent. Cochrane Database Syst Rev. 2017;12(12):CD011300. doi:10.1002/14651858.CD011300.pub2

\section{Publish your work in this journal}

Cancer Management and Research is an international, peer-reviewed open access journal focusing on cancer research and the optimal use of preventative and integrated treatment interventions to achieve improved outcomes, enhanced survival and quality of life for the cancer patient.
The manuscript management system is completely online and includes a very quick and fair peer-review system, which is all easy to use. Visit http://www.dovepress.com/testimonials.php to read real quotes from published authors. 